\title{
Advanced students' and faculty members' reasoning about the double slit experiment with single particles
}

\author{
Ryan Sayer \\ Department of Physics, Bemidji State University, Bemidji, MN, 56601, USA \\ Alexandru Maries ${ }^{1}$, and Chandralekha Singh ${ }^{2}$ \\ ${ }^{1}$ Department of Physics, University of Cincinnati, Cincinnati, OH 45221, USA \\ ${ }^{2}$ Department of Physics and Astronomy, University of Pittsburgh, Pittsburgh, PA, 15260, USA
}

\begin{abstract}
We describe an investigation focusing on advanced students' and faculty members' reasoning about two questions related to the double-slit experiment with single particles. One question posed was a standard double slit question while the other question was more speculative. First, students in advanced quantum mechanics courses were asked the questions in written form and individual interviews were conducted with six students in which they were asked follow up questions to make their thought processes explicit. We also interviewed five faculty members who had taught modern physics, quantum mechanics and/or solid state physics to understand their reasoning and thought processes. The faculty members' responses shed light on what it means to think like a physicist. Student responses varied greatly in their correctness and sophistication of reasoning, suggesting that even advanced students need scaffolding support in order to learn to think like a physicist.
\end{abstract}




\section{INTRODUCTION AND GOAL}

The double slit experiment (DSE) with single particles has played a seminal role in demonstrating the wave nature of particles in quantum mechanics (QM). The DavissonGermer experiment in the 1920s, in which slow moving electrons were fired at a crystalline nickel target, confirmed the de Broglie hypothesis pertaining to the wave-particle duality; the angular dependence of the reflected electron intensity and diffraction patterns were similar to those predicted for X-rays [1]. Due to experimental challenges, a single-electron DSE which shows the buildup of the interference pattern and also confirms the wave nature of electrons was demonstrated much later in 1974, when single electrons were passed through a biprism (an electron optical device) that serves the same function as a double slit [2]. Since then, the DSE has even been conducted with larger molecules, for example, buckyballs $\left(\mathrm{C}_{60}\right.$ molecules) were sent one at a time under controlled conditions to minimize decoherence of the system, and an interference pattern was observed, confirming the wave nature of particles [3]. Currently, there is effort to investigate whether decoherence in the context of the DSE can be minimized to the extent that an interference pattern can be observed with living things such as viruses and bacteria.

Prior research in student learning of QM, e.g., see Ref. [4-36], demonstrates that developing expertise in the context of advanced QM is particularly challenging, and the patterns of difficulties in the context of QM bear a striking resemblance to those found in introductory classical mechanics [20]. These analogous patterns of difficulties are often due to the diversity in the goals and prior preparation of upper-level students [37], as well as the "paradigm shift" from classical mechanics to QM. In particular, developing expertise and learning to think like a physicist in the context of QM can be especially challenging for students because the paradigms of classical mechanics and QM are very different [20]. For example, in classical physics position and momentum are deterministic variables, whereas in QM they are operators that act on a wave function (or a state) which lies in an abstract Hilbert space. In addition, according to the Copenhagen interpretation, the most commonly taught interpretation in QM courses, an electron does not, in general, have a definite position; it is the act of measurement that collapses the wave function and makes it localized. If the wave function is known right before the measurement, quantum theory only provides the probability of measuring the electron's position within a given range. Due to the significantly different paradigms of classical mechanics and QM, even students with a good knowledge of classical mechanics will start learning QM as novices. They will benefit from scaffolding support as they develop expertise and gradually build a good knowledge structure of QM.

Here, we describe an investigation focusing on advanced students' and faculty members' understanding and reasoning about the DSE with single particles, which could be useful as a guide for creating learning tools to help students develop expert-like reasoning about these challenging QM concepts. We note that this is part of a larger investigation, and we restrict our attention here to students' and faculty members' responses to two questions. One of the questions focuses on how the interference pattern in the DSE changes when one particle is replaced with another particle with larger mass while the other experimental parameters, such as kinetic energy, slit separation, and slit width, are kept fixed (i.e., the de Broglie wavelengths of the particles are different but the setup is otherwise the same). The other question is more speculative and focuses on reasoning and arguments for why a DSE will or will not show an interference pattern when macroscopic entities such as sand particles are sent one at a time through the slits with well-chosen parameters.

\section{METHODOLOGY}

Our investigation started with a survey of prior research on student understanding of the DSE in the context of both modern physics and QM courses. Prior studies have found that students have great difficulty with the wave-particle duality, generally favoring the particle nature, and sometimes attempt to reconcile the wave model with the particle model by commenting that an electron (which they view as a localized particle) moves sinusoidally like a wave [4]. Another study suggests that when explaining the pattern observed in a DSE involving particles with mass, students often use classical concepts and state that the pattern is a result of the particles bouncing against one another in the experiment [5]. Even when explaining the pattern in terms of interference, students often do so by stating that one electron emerging from one slit interferes with another electron from the other slit [6,7]. Vokos et al. developed a tutorial on the wave properties of matter which was found to be effective in improving student understanding of matter-wave duality [7].

We developed and validated open-ended questions which build on prior research on student difficulties with the DSE and administered them to advanced undergraduate and graduate students enrolled in various QM classes. Based upon student responses to questions in earlier QM courses, some of the questions on the DSE were revised. The two questions discussed in this study went through several iterations and modifications.

The undergraduate students answered the questions as a quiz in a required advanced undergraduate QM course after instruction in the relevant concepts: the de Broglie relation, calculation of the de Broglie wavelengths of different particles, an overview of the patterns that form on the distant screen in the DSE after a large number of single particles are sent one at a time through the slits, and a brief overview of the relevance of the information about which slit the particle went through to whether an interference pattern is observed on the screen. The graduate students answered these 
questions without prior instruction in their course to investigate what they may have learned and remembered from their previous undergraduate courses. The questions were graded using a rubric by two investigators. After comparing the grading of some students, the raters discussed any disagreements and resolved them so that the interrater agreement was better than $90 \%$.

Individual interviews were conducted with six students and five faculty members using a semi-structured, thinkaloud protocol to provide the researchers with a deeper understanding of the rationale used to answer foundational questions related to the DSE. During the semi-structured interviews, advanced undergraduate and graduate students (same institution) and faculty were asked to verbalize their thought processes while answering the questions. They first read the questions and then answered them to the best of their ability without being disturbed. They were prompted to think aloud if they became quiet for a long time. After they had finished answering a particular question to the best of their ability, they were often asked to further clarify and elaborate issues that they had not addressed earlier.

Before interviews with students and faculty members, the two questions discussed here were first posed as written inclass quiz questions to 46 undergraduate students in junior/ senior level QM courses and 45 graduate students enrolled in core graduate QM courses after traditional instruction in the relevant concepts. The quiz was graded for correctness (undergraduate QM) or completeness (graduate QM).

In the first question, one sends a large number of nonrelativistic electrons of the same kinetic energy one at a time towards a double-slit plate and observes an interference pattern. Students are asked how the pattern on the screen would change, if at all, if the experiment is modified by using protons instead of electrons while all of the following parameters are held fixed: kinetic energy, slit width, slit separation, and distance between the slits and screen. The second, more speculative question posed in this research was the following: "Consider particles of sand, which can be approximated as spheres of a radius of about $1 / 10$ of a millimeter. Do you expect that a double slit experiment with well-chosen parameters would show an interference pattern? Explain your reasoning."

\section{RESULTS}

The written responses of undergraduate and graduate students to question 1 are shown in Table I. The correct response is that the distance between interference fringes will become narrower since the de Broglie wavelength of a proton is shorter than the wavelength of an electron if the particles have the same kinetic energy. One third of the undergraduates and a quarter of the graduate students provided the correct response. One possible reason for the lower performance of the graduate students is that they did not have instruction in these concepts in the graduate course where the quiz was administered (unlike the undergraduates).
In this question related to how the change in the input (changing the mass of the particle) affects the output (interference pattern), there is a strong resource or p-prim $[38,39]$ that students use, i.e., a change in the input must result in a change in the output. In Table I, responses based upon this p-prim would correspond to responses in the categories A) fringe spacing decreases, B) fringe spacing increases, C) there must be a change, but unsure about how the fringe spacing would change, and F) in this new situation, there won't be interference. Table I shows that this p-prim was strong. Approximately two-thirds of the responses fall in these categories and, excluding the "no-interference" case, $50 \%$ of the student responses for both undergraduates and graduate students fall in categories A), B) and C), in which A) is the correct response. Some students who responded that the spacing between fringes will become larger claimed that the wavelength increases with mass, when in fact the wavelength will be shorter for more massive particles if the particles have the same kinetic energy.

Another common p-prim is that if some salient input parameters do not change, the output must remain unchanged. Table 1 shows that a quarter of the undergraduates and graduate students used this p-prim and claimed that there would be no change because the kinetic energy of the particles and the slit width and distances are unchanged. Written reasoning and interviews corroborate that many students incorrectly assumed that if two particles of different types (e.g., masses) have the same kinetic energy then they must have the same wavelength, so the distance between fringes would not change when the slit parameters are also unchanged.

For the second question, student reasoning was the main criterion used to determine correctness. For example, students who noted that they do NOT expect to observe an interference pattern regardless of the experimental parameters were counted as correct if they noted that the experimental parameters for this case are unrealistic. These students generally estimated the de Broglie wavelength for the sand particle and noted that interference is not possible because the wavelength of the sand particles is orders of magnitude smaller than any physical setup for a double slit that can be constructed realistically to observe interference (e.g., how could the sand particles pass through a physical slit which is of the order of its de Broglie wavelength if the distance between the slits is also of the same order?). They understood that all particles have an associated wavelength and focused on the fact that the parameters for the DSE with

TABLE I. Percentages of undergraduate (upper row) and graduate student (lower row) written responses to question 1 (classifications in categories A, B, C, F refer to those who predicted a change).

\begin{tabular}{|c|c|c|c|c|c|c|}
\hline $\begin{array}{c}\text { A: Fringe } \\
\text { spacing } \\
\text { decreases }\end{array}$ & $\begin{array}{c}\text { B: Fringe } \\
\text { spacing } \\
\text { increases }\end{array}$ & $\begin{array}{c}\text { C: some } \\
\text { type of } \\
\text { Change }\end{array}$ & $\begin{array}{c}\text { D: No } \\
\text { Change }\end{array}$ & $\begin{array}{c}\text { E: } \\
\text { Inter- } \\
\text { ference }\end{array}$ & $\begin{array}{c}\text { F: No } \\
\text { Inter- } \\
\text { ference }\end{array}$ & $\begin{array}{c}\text { G: Other } \\
\text { /No } \\
\text { Response }\end{array}$ \\
\hline 34 & 14 & 2 & 25 & 5 & 14 & 7 \\
\hline 25 & 14 & 11 & 25 & 5 & 18 & 2 \\
\hline
\end{tabular}


sand particles with very small wavelength could not be achieved in a realistic situation: the large sand particles would be blocked by the narrow slits of the order of their de Broglie wavelength even if such a slit could be constructed.

Furthermore, since an equivalent experiment to that of a DSE with sand particles would require that the distance between the slits and slit widths be comparable to the de Broglie wavelength of the sand particles, which is very small, one needs to consider how to physically design an experiment that is equivalent to the DSE without using physical slits since the sand particles are large. Although we would have counted student responses to be correct if they had noted that such an experiment could at least be conceived if one could achieve the conditions for a DSE using some clever technique for sand particles with such small de Broglie wavelength without explicitly using physical slits (although such an experiment is not envisioned any time in the near future), no student provided this type of response.

Overall, roughly $45 \%$ of the undergraduate and graduate students answered this question correctly, and there was no difference between the two groups. The most common incorrect student response was to not focus on the de Broglie wavelength of the sand particles and only focus on their size. For example, some students who provided an incorrect response claimed that one needs slits with a size larger than that of the sand particles so that sand particles can pass through the slits in order to observe interference.

In individual interviews, in response to the first question, one student who provided an incorrect response stated:

"This has me thinking about what the wavelength is because I know that there is dependence of fringes on wavelength... The de Broglie wavelength is dependent on kinetic energy so if they both have the same kinetic energy then I believe they would have the same interference fringes."

On the other hand, all faculty members provided correct responses to question 1 . In an individual interview, one faculty member stated:

"If the kinetic energy is the same, mass increases of proton 2000 times which means the momentum increases by square root of that. The wavelength goes down by a factor of thirtyish. Oh I have not done this in a while (laughs) So if that is what happens then I think the interference pattern just gets tighter, thirty times tighter on the screen-everything gets closer."

In response to the second question, which was more speculative, one interviewed faculty member responded:

"The answer is "no" for any reasonable experiment you set up because of the size of the mass. You basically have to calculate the wavelength, which, if you pick the energy, can be calculated... you will get a really really tiny wavelength because you have a very big mass here. And hence the pattern shrinks to be unobservable."

The two comments by the faculty members suggest that they have a lot of compiled knowledge (e.g., wavelength of proton is unobservable), but since they also know where that knowledge is coming from, they can easily recreate it if they forgot it (in this case, by using the de Broglie relation).

Regarding question 2, another interviewed faculty stated ( $\mathrm{I}=$ interviewer, $\mathrm{FM}=$ faculty member $)$ :

FM: "I don't think I would. $1 / 10^{\text {th }}$ of mm-that is 100 micrometers. This is of the order of $10^{20}$ protons. That is how big you expect the sand particle to be... The mass of the sand particle is about $10^{20}$ larger than proton... The short answer is no. realistically no... it would have to be the wavelength lambda divided by slit width times the separation to the screen... If the fringes show up that would be roughly the order of the distance you will expect. First of all this number is going to be ridiculously small... Suppose you manage to achieve all the criteria for quantum coherence in a double slit experiment which means that it is cold enough that you manage to keep quantum coherence, forget about the internal degrees of freedom, hypothetically we will be able to do all of that, even then your distance between fringes will be so small that optically the fringes won't be resolvable...Oh I forgot I guess in double slit, the distance between the slits will be dominant one but on top of that there is single slit pattern....The other thing is that normally you wouldn't expect it to be quantum coherent because each sand particle consists of so many internal particles that you also need to make it really really cold so that you treat it as one quantum particle as opposed to a many body system to actually get interference so everything will be $10^{20}$ bigger and $10^{20}$ harder [...] to do this experiment because the de Broglie wave length will be $10^{20}$ smaller than in the previous case." I: What will happen if you let the particles go through the slit? FM: "It won't even fit through the slit, so you will need quantum tunneling on top of that in order to make it work." I: What will happen if slit width is of the size of the particles? FM: "Well, in an actual double slit or single slit experiment... that would make the interference pattern even smaller... You will run into more practical problems. Having a big slit means that you will have more practical problems..."

I: Can you have the de Broglie wavelength be smaller than the size of the object?

FM: "Yes...well it does not mean you will not see interference pattern. It just means it is going to be that much harder to see it. Like there is no sharp cutoff that if the de Broglie wavelength is smaller than the separation of atoms in the sand then you will not see interference pattern. But the short answer is that you should treat it more classically than quantum. I imagine the visibility will be heavily suppressed to the point that it would be impossible to see interference... I am saying you should not be able to see patterns because the numbers are such that it is not practical to see double slit pattern. It does not mean quantum mechanics does not apply to particles of this size. It is just that you do not expect to observe any quantum interference at all-you would expect to see the same result as if you had done the calculation classically which means in your case it will just go through one of the slits... it will be unresolvable." 
This interview with the faculty member suggests that he was thinking like a physicist and was resorting to metacognition in order to think through the situation on his feet when probed further. He was able to resort to his knowledge of physics as appropriate to refine his arguments.

Another interviewed faculty first brought up similar issues stating that a sand particle is macroscopic and essentially behaves classically due to decoherence. However, he stated that assuming we ignore the decoherence effects and pretend that we can treat the sand particles quantum mechanically:

"then interference effects will depend on the de Broglie wavelength which will be incredibly small for a grain of sand. So small that you will never be able to construct a grating. The minimum size of the grating will be the size of the slit so you can calculate basically where the first order diffraction peak will be and what the angle will be. And my guess is that you would not resolve it within the size of the universe. [...] Let me estimate the de Broglie wavelength for a sand particle moving at a speed of $100 \mathrm{~m} / \mathrm{s}$. If the mass of the sand particle is $10^{-5} \mathrm{~kg}$ then its momentum is $p=m v=$ $10^{-3} \mathrm{~kg} \mathrm{~m} / \mathrm{s}$. So the de Broglie wavelength is $\lambda=h / p=6 \times$ $10^{-34} \mathrm{~J} \mathrm{~s} / 10^{-3} \mathrm{~kg} \mathrm{~m} / \mathrm{s}=10^{-30} \mathrm{~m}$."

The faculty member then drew a diagram showing a point on the distant screen and connected it to the center of the two slits and drew an angle theta for the angle between them and stated that:

"in order to see first order fringe on a very distant screen, $d \sin \theta=\lambda$ or $d \theta=\lambda$ where let's assume that the distance between the slits $d$ is of the order of the size of the sand particle $10^{-4} \mathrm{~m}$. Note that if we make d larger than this, the angle theta will be even smaller and resolving two bright fringe on the screen will become even more difficult... So the angle $\theta=\lambda / d=10^{-26}$. The question is... how far should the screen be where interference fringes are observed so that two adjacent bright fringes, say zeroth order and first order can be resolved? The distance between adjacent fringes should be at the very least of the order of the size of the sand particles $10^{-4} \mathrm{~m}$ so the minimum distance of the screen from the two slits in order to resolve it should be $L=10^{-4} \mathrm{~m} / \theta$ $=10^{-4} \mathrm{~m} / 10^{-26}=10^{22} \mathrm{~m}$."

The faculty member then estimated the size of the universe by using its age and the speed of light to obtain $\sim 10^{26} \mathrm{~m}$. He then went on to say:

" $10^{-4}$ times size of the universe, -it is a very very big distance. I am not an astrophysicist, but people work in astronomical unit which is the distance between earth and sun... so 1/10000 times the size of the universe sounds small but it is very very large distance that is not practical for doing a double slit experiment with distant screen. The problem is that we have a universe filled with objects and radiation and those are going to distinguish between different paths because the universe is not empty. There is no way you will be able to do this experiment with parameters of the sand and see interference patterns".
This interview also displays how the faculty member used the problem to think like a physicist. His metacognitive process focused on showing that it is not possible to resolve the fringes within the size of the universe. Other faculty members' responses were also very reflective.

\section{DISCUSSION AND SUMMARY}

The part of a larger investigation described here focuses on advanced students' and faculty members' responses and reasoning about two questions pertaining to the DSE with single particles, a counterintuitive experiment that contradicts classical notions of particles and waves. In the first question posed, many students employed p-prims (e.g., if kinetic energy does not change, the interference pattern won't change). In the more speculative question posed, macroscopic sand particles were sent through the slits and people were asked to explain whether interference can be observed under suitable conditions in this situation. All faculty members' responses demonstrated what it means to think like a physicist even in a situation which required thinking on their feet. In contrast, the student responses varied greatly in their correctness, coherence and sophistication of reasoning. While some students provided expert-like reasoning similar to faculty even in this nonintuitive context, others struggled.

The sophistication of all interviewed faculty members' responses suggests that even in this counterintuitive context, physics experts can reason systematically using their knowledge structure and the demonstration of their reasoning and metacognitive skills sheds light on the fact that even in this context of QM we should strive to help students develop a similar level of expertise. Furthermore, while the diversity in different students' preparation, goals, and motivations may partly account for the diversity in student responses, student difficulties in the challenging context of the DSE may be exacerbated by the fact that the paradigm of QM is significantly different than the classical physics paradigm that students have previously learned and internalized. In particular, the very nature of a new paradigm causes additional difficulties even for many advanced students in learning to think like a physicist in the challenging context of QM.

However, the importance of QM is only likely to increase in the future, e.g., with quantum information science and technology becoming increasingly important for solving major societal, national security, and economic problems. Therefore, helping students with diverse prior preparation build a good knowledge structure of QM and learn to reason like an expert is of paramount importance. The findings of this research can be useful for contemplating pedagogies to help students in QM courses learn to think like a physicist and develop expert-like problem solving, reasoning and metacognitive skills.

\section{ACKNOWLEDGMENTS}

We thank the NSF for award PHY 1806691. 
[1] C. Davisson and L. Germer, Reflection of electrons by a crystal of Nickel, Proc. Nat. Acad. Sci., 14, 317 (1928).

[2] P. Merli, G. Missiroli and G. Pozzi, On the statistical aspect of electron interference phenomena, Am. J. Phys. 44, 306 (1976).

[3] M. Arndt, O. Nairz, J. Vos-Andreae, C. Keller, G. van der Zouw and A. Zeilinger, Wave-particle duality of $\mathrm{C}_{60}$ molecules, Nature 401, 680 (1999).

[4] A. Mashhadi, Students' conceptions of quantum physics, in Thinking Physics for Teaching, edited by C. Bernardini, C. Tarsitani, and M. Vicentini (Plenum Press, New York, NY 1995), p. 313.

[5] B. Thacker, A study of the nature of students' models of microscopic processes in the context of modern physics experiments, Am. J. Phys 71, 599 (2003).

[6] B. Ambrose, P. Shaffer, R. Steinberg, and L. McDermott, An investigation of student understanding of single-slit diffraction and double-slit interference, Am. J. Phys. 67, 146 (1999).

[7] S. Vokos, P. Shaffer, B. Ambrose, and L. McDermott, Student understanding of the wave nature of matter: Diffraction and interference of particles, Am. J. Phys. 68, S42 (2000).

[8] C. Singh, Student understanding of quantum mechanics, Am. J. Phys. 69, 885 (2001).

[9] D. Zollman, N. Rebello and K. Hogg, Quantum mechanics for everyone: Hands-on activities integrated with technology, Am. J. Phys. 70, 252 (2002).

[10] M. Wittmann, R. Steinberg, and E. Redish, Investigating student understanding of quantum physics: Spontaneous models of conductivity, Am. J. Phys. 70, 218 (2002).

[11] C. Singh, Interactive learning tutorials on quantum mechanics, Am. J. Phys. 76, 400 (2008).

[12] G. Zhu and C. Singh, Improving students' understanding of quantum mechanics via the Stern-Gerlach experiment, Am. J. Phys. 79, 499 (2011).

[13] E. Gire and C. Manogue, Resources students use to understand quantum mechanical operators in Proceedings of the Physics Education Research Conference, AIP Conf. Proc., Melville, NY (2012).

[14] G. Zhu and C. Singh, Surveying students' understanding of quantum mechanics in one spatial dimension, Am. J. Phys. 80, 252 (2012).

[15] G. Zhu and C. Singh, Improving students' understanding of quantum measurement: I. Investigation of difficulties, Phys. Rev. ST PER 8, 010117 (2012).

[16] G. Zhu and C. Singh, Improving students' understanding of quantum measurement: II. Development of research-based learning tools, Phys. Rev. ST PER 8, 010118 (2012).

[17] G. Zhu and C. Singh, Improving student understanding of addition of angular momentum in quantum mechanics, Phys. Rev. ST PER 9, 010101 (2013).

[18] A. Kohnle I. Bozhinova, D. Browne, M. Everitt, A. Fomins, P. Kok, G. Kulaitis, M. Prokopas, D. Raine and E. Swinbank, A new introductory quantum mechanics curriculum, Eur. J. Phys. 35, 015001 (2014).

[19] C. Singh and E. Marshman, Review of student difficulties with quantum mechanics, Phys. Rev. ST Phys. Educ. Res. 11, 020117 (2015)

[20] E. Marshman and C. Singh, Framework for understanding student difficulties in quantum mechanics, Phys. Rev. ST Phys. Educ. Res. 11, 020119 (2015).

[21] E. Gire and E. Price, The structural features of algebraic quantum notations, Phys. Rev. ST Phys. Educ. Res. 11, 020109 (2015).

[22] P. Emigh, G. Passante, and P. Shaffer, Student understanding of time dependence in quantum mechanics, Phys. Rev. ST Phys. Educ. Res. 11, 020112 (2015).

[23] E. Marshman and C. Singh, Interactive tutorial to improve student understanding of single photon experiments involving a Mach-Zehnder Interferometer, Eur. J. Phys. 37, 024001 (2016).

[24] R. Sayer, A. Maries and C. Singh, A quantum interactive learning tutorial on the double-slit experiment to improve student understanding of quantum mechanics, Phys. Rev. Phys. Educ. Res. 13, 010123 (2017).

[25] V. Dini and D. Hammer, Case study of a successful learner's epistemological framings of quantum mechanics, Phys. Rev. Phys. Educ. Res. 13, 010124 (2017).

[26] R. Sayer, A. Maries and C. Singh, A quantum interactive learning tutorial on the double-slit experiment to improve student understanding of quantum mechanics, Phys. Rev. PER 13, 010123 (2017).

[27] E. Marshman and C. Singh, Investigating and improving student understanding of the probability distributions for measuring physical observables in quantum mechanics, Eur. J. Phys. 38, 025705 (2017).

[28] E. Marshman and C. Singh, Investigating and improving student understanding of quantum mechanics in the context of single photon interference, Phys. Rev. PER 13, 010117 (2017).

[29] C. Keebaugh, E. Marshman and C. Singh, Improving student understanding of corrections to the energy spectrum of the hydrogen atom for the Zeeman effect, Phys. Rev. Phys. Educ. Res. 15, 010113 (2018).

[30] E. Marshman and C. Singh, Investigating and improving student understanding of quantum mechanical observables and their corresponding operators in Dirac notation, Eur. J. Phys. 39, 015707 (2018).

[31] C. Keebaugh, E. Marshman and C. Singh, Investigating and addressing student difficulties with a good basis for finding perturbative corrections in the context of degenerate perturbation theory, Eur. J. Phys. 39, 055701 (2018).

[32] C. Keebaugh, E. Marshman and C. Singh, Investigating and addressing student difficulties with the corrections to the energies of the hydrogen atom for the strong and weak field Zeeman effect, Eur. J. Phys. 39, 045701 (2018).

[33] C. Keebaugh, E. Marshman and C. Singh, Improving student understanding of fine structure corrections to the energy spectrum of the hydrogen atom, Am. J. Phys. 87, 594 (2019).

[34] C. Keebaugh, E. Marshman and C. Singh, Improving student understanding of a system of identical particles with a fixed total energy, Am. J. Phys. 87, 583 (2019).

[35] E. Marshman and C. Singh, Validation and administration of a conceptual survey on the formalism and postulates of quantum mechanics, Phys. Rev. PER 15, 020128 (2019).

[36] S. Devore and C. Singh, An interactive learning tutorial on quantum key distribution, Phys. Rev. PER 16, 010126 (2020).

[37] AIP Statistical Research Center http://www.aip.org/statistics

[38] D. Hammer, Misconceptions or p-prims: How may alternative perspectives of cognitive structure influence instructional perceptions and intentions, J. Learn. Sci. 5, 97 (1996).

[39] A. diSessa, "Knowledge in Pieces" in Constructivism in the Computer Age, Lawrence Erlbaum Publishers (1988). 